\title{
乌ु \\ Early transverse decoherence of bunches with space charge
}

\author{
Ivan Karpov, ${ }^{1}$ Vladimir Kornilov, ${ }^{2}$ and Oliver Boine-Frankenheim ${ }^{1,2}$ \\ ${ }^{1}$ Technische Universität Darmstadt, Schlossgartenstraße 8, 64298 Darmstadt, Germany \\ ${ }^{2}$ GSI Helmholtzzentrum für Schwerionenforschung, Planckstraße 1, 64291 Darmstadt, Germany
}

(Received 24 June 2016; published 27 December 2016)

\begin{abstract}
The transverse decoherence of injected bunches is an important phenomenon in synchrotrons and storage rings. The initial stage of this process determines the transverse emittance blowup, which should be taken into account for the design of feedback systems, for example. The interplay of different high-intensity effects can strongly affect the initial decoherence stage. We present a model that explains decoherence and emittance growth with chromaticity, space charge, and image charges within the first synchrotron period. We compare the model for different combinations of parameters with self-consistent particle tracking simulations and measurements in the SIS18 synchrotron at GSI Darmstadt. Generally, space charge slows down the decoherence process and can cause the loss of decoherence. Chromaticity and image charges can partly compensate this loss and restore the decoherence. We also analyze the single-particle excitation driven by space charge during the decoherence process. Particles gain large amplitudes from the coherent beam oscillation, which leads to halo buildup and losses.
\end{abstract}

DOI: 10.1103/PhysRevAccelBeams.19.124201

\section{INTRODUCTION}

After an initial transverse offset the amplitude of the beam centroid oscillations decays due to the spread of individual particle frequencies. This process is usually called decoherence or phase-mixing. Transverse decoherence can be used for diagnostic purposes (e.g., for chromaticity measurements [1]), but it can also reduce the machine performance. For example, after the bunch-to-bucket transfer in synchrotrons decoherence results in a transverse emittance blowup. In order to prevent beam quality degradation a transverse feedback system (TFS) should be used with a damping time shorter than the characteristic decoherence time. In the presence of transverse nonlinearities and chromaticity the time evolution of the bunch offset and of the rms emittance were obtained analytically in Refs. [2,3].

Intensity effects significantly change the decoherence because of additional incoherent and coherent tune shifts. Attempts to understand the decoherence process and the emittance growth with space charge and impedances were presented in Refs. [4-10]. The role of space charge and its interplay with other intensity effects, which is important for the design of the SIS100 synchrotron as part of the FAIR project [11], still needs to be clarified.

Recently, a different approach was applied to describe the long-term behavior of decoherence and recoherence following a small initial offset $[12,13]$. The initial kick was represented as a combination of bunch head-tail

Published by the American Physical Society under the terms of the Creative Commons Attribution 3.0 License. Further distribution of this work must maintain attribution to the author(s) and the published article's title, journal citation, and DOI. eigenmodes, which are prone to different Landau damping rates. The approach predicts that after a transition time a mixture of the remaining eigenmodes continues to oscillate, exhibiting a characteristic time pattern. The initial stage of the decoherence process in intense bunches affected by space charge for arbitrary offsets is the subject of the present work. We study the interplay of space charge, chromaticity, and image charges during the decoherence process.

In Sec. II a brief discussion of the decoherence and the emittance growth in the absence of intensity effects is presented. The effect of space charge on the decoherence process is discussed in Sec. III. We develop an analytical model to describe decoherence in coasting beams. The model is verified by particle tracking simulations with the PATRIC code [14]. The resonant excitation of particles driven by the coherent beam oscillations is discussed in Sec. III B. It is shown that those particles can contribute to losses and halo buildup. In Sec. III C we apply the extended model to the initial stage of decoherence in ion bunches. In Sec. IV we discuss the contribution of image charges. Comparisons with measurements performed in the SIS18 synchrotron [15] at GSI Darmstadt are presented in Sec. V. For the intensities achieved in the experiments it is shown that the decoherence is governed by chromaticity and space charge. The work is concluded in Sec. VI.

\section{DECOHERENCE WITHOUT SPACE CHARGE}

The analytical calculation of the bunch offset evolution in Refs. [2,3] assumes that at a certain time all particles experience the same transverse kick independent of their longitudinal position. For each time step the transverse 
bunch offset is calculated as the average of all particle coordinates. We call this a fixed-time monitoring (ft) [16].

Considering the case of linear synchrotron motion, linear chromaticity $\xi=\frac{\Delta Q}{Q_{0}} / \frac{\Delta p}{p_{0}}$, and a Gaussian momentum distribution, the amplitude of beam oscillations $A$ after a transverse kick is given by [2]

$$
\frac{A(N)}{A_{0}}=\exp \left\{-2 \frac{\delta Q_{\xi}^{2}}{Q_{s}^{2}} \sin ^{2}\left(\pi Q_{s} N\right)\right\}
$$

Here, $N$ is the time variable expressed in the number of turns, $A_{0}$ is the initial beam oscillation amplitude after the kick, $Q_{s}$ is the synchrotron tune, and the chromaticity tune spread,

$$
\delta Q_{\xi}=|\xi| Q_{0} \frac{\delta p}{p_{0}}
$$

where $\delta p / p_{0}$ is the normalized rms momentum spread and $Q_{0}$ is the bare betatron tune.

During the first half of the synchrotron period $N_{s}=1 / Q_{s}$, the chromaticity tune spread leads to mixing of particle betatron phases and we observe decoherence. After the synchrotron period, the betatron phase spread is compensated and the beam oscillation amplitude returns to the initial value. This process is called recoherence.

The first and the second moments of the transverse distribution as functions of time were calculated in Refs. [3,17]. These can be used to calculate the time evolution of the rms emittance $\epsilon$. An approximate equation for the emittance blowup is given by [3]

$$
\frac{\Delta \epsilon(N)}{\epsilon_{0}}=\frac{\epsilon(N)-\epsilon_{0}}{\epsilon_{0}} \approx \frac{1}{2} \frac{A_{0}^{2}}{\sigma_{0}^{2}}\left(1-\frac{A^{2}(N)}{A_{0}^{2}}\right),
$$

which shows a simple dependence on the beam oscillation amplitude. Here $\epsilon_{0}$ is the initial rms emittance of the beam, $\sigma_{0}$ is the initial rms beam size at the position of the kicker. The maximum emittance growth is

$$
\Delta \epsilon_{\max }=\frac{\epsilon_{0} A_{0}^{2}}{2 \sigma_{0}^{2}},
$$

and it does not depend on the transverse distribution (see, e.g., in Ref. [18]).

In a real situation the kicker applies a kick in time at the fixed location. The initial betatron phase of the particle oscillation depends on the particle's longitudinal position. After the kick we observe the signals in time using a beam position monitor (BPM) at a fixed position in the ring. This we call a fixed-position monitoring (fp) [16]. In this case, the observed decoherence is different form the fixed-time monitoring and Eq. (1) is not valid. Particles pass by the $\mathrm{BPM}$ with different time delays $\Delta T / T_{0}=\eta \Delta p / p_{0}$ due to

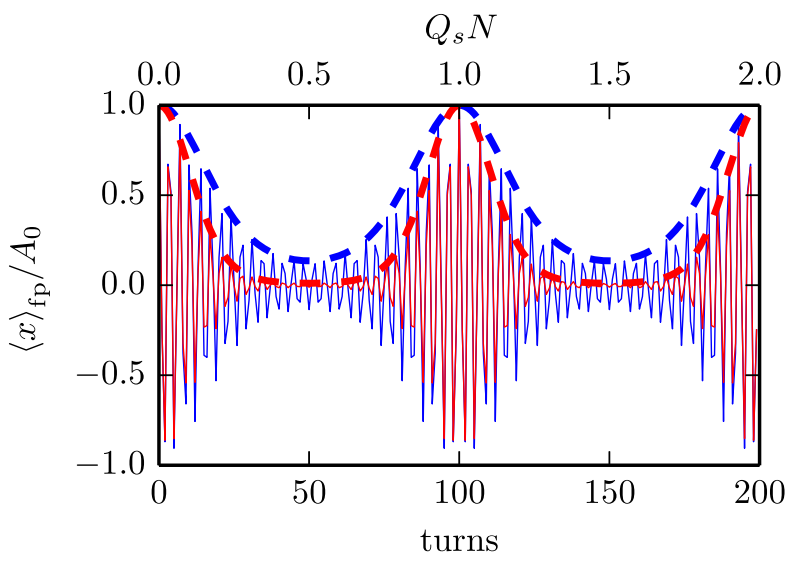

FIG. 1. Time evolution of the beam offset after the transverse kick from particle tracking simulations with the fixed-position monitoring for $Q_{s}=0.01$. Red lines are for $\delta Q_{\xi}=0.01$ and $\delta Q_{\text {eff }}=0.015$, blue lines are for $\delta Q_{\xi}=0.005$ and $\delta Q_{\text {eff }}=0.01$ $(\xi<0$ and $\eta<0)$. The solid lines are the bunch offsets and the dashed lines are given by Eq. (1) with the effective tune spread from Eq. (5).

different momenta. Additionally to the chromaticity tune spread there is a contribution from the slip factor $\eta$,

$$
\delta Q_{\mathrm{eff}}=|\eta+\xi| Q_{0} \frac{\delta p}{p_{0}}
$$

which we call the effective tune spread.

Both ways of monitoring the beam properties are equivalent if we substitute $\delta Q_{\xi}$ in Eq. (1) by $\delta Q_{\text {eff }}$ for the fixed-position monitoring. We demonstrate this in particle tracking simulations obtained by the PATRIC code [14]. Without intensity effects, the code employs a simple tracking in the constant-focusing lattice with the linear synchrotron motion. A bunch with a round cross section has Gaussian transverse and longitudinal profiles. The transverse distribution is truncated at $3.5 \sigma_{0}$, and the momentum distribution is truncated at $3 \delta p / p_{0}$. The oscillation amplitude given by Eq. (1) where $\delta Q_{\xi}$ is substituted by $\delta Q_{\text {eff }}$ perfectly agrees with simulation results (Fig. 1 ).

In this paper we use a constant-focusing approximation of betatron oscillations for analytic and simulation studies. Below we present simulations with the fixedtime monitoring while using the notation of the tune spread given by Eq. (5) for direct comparisons with the measurements.

\section{DECOHERENCE WITH SPACE CHARGE}

For the description of transverse space charge we use the characteristic tune shift,

$$
\Delta Q_{\mathrm{sc}}=\frac{\lambda_{0} r_{p} C}{8 \pi \gamma^{3} \beta^{2} \epsilon}
$$


Here, $\lambda_{0}$ is the peak line density, $r_{p}=q_{\mathrm{ion}}^{2} /\left(4 \pi \varepsilon_{0} m_{\text {ion }} c^{2}\right)$ is the classical particle radius, $q_{\text {ion }}$ and $m_{\text {ion }}$ are the charge and the mass of the ions, and $C$ is the machine circumference. The parameter $\Delta Q_{\mathrm{sc}}$ corresponds to the modulus of the negative tune shift for the rms-equivalent beam with a round cross section and a Kapchinskij-Vladimirskij (K-V) transverse distribution. For nonuniform transverse and longitudinal beam profiles, space charge also creates a tune spread. For a Gaussian transverse distribution, $\Delta Q_{\mathrm{sc}}$ estimated by Eq. (6) is half of the maximum tune shift, and it is close to the average tune shift of all particles [19]. The additional tune shift and the tune spread change single particle dynamics as well as coherent beam dynamics. Thus decoherence with space charge is very different from the low-intensity case $[4-8,12,13]$.

The initial stage of decoherence-the first synchrotron period-is decisive for the transverse emittance blowup. As the first step, we consider the 2D case of a coasting beam.

\section{A. 2D case "coasting beam"}

Decoherence of a coasting beam can be described using the pulse response function $[19,20]$. For the case of the linear chromaticity and a Gaussian momentum spread, the pulse response function is

$g_{0}(N)=\frac{\langle x\rangle}{A_{0}}=\exp \left\{-2\left(\pi \delta Q_{\text {eff }} N\right)^{2}\right\} \cos \left(2 \pi Q_{0} N\right) H(N)$,

where $H(N)$ is the Heaviside step function. We define the characteristic decoherence time

$$
N_{\mathrm{dec} 0}=\left(\sqrt{2} \pi \delta Q_{\mathrm{eff}}\right)^{-1},
$$

which corresponds to the $1 / e$-decrease of the oscillation amplitude. The Fourier transform of the pulse response function $\mathcal{F}\left\{g_{0}\right\}$ is proportional to the beam transfer function (BTF),

$$
r_{0}(Q)=\sqrt{\frac{\pi}{2}} w\left(\frac{Q_{0}-Q}{\delta Q_{\mathrm{eff}} \sqrt{2}}\right),
$$

where $w(z)=\exp \left(-z^{2}\right)[1-\operatorname{erf}(-i z)]$ is the complex error function. The real and the imaginary part of BTF for the considered case are related by the Hilbert transform [20]. The plot of the inverse BTF in the complex plane is called stability diagram [21]. The modification of the BTF due to space charge was discussed in Refs. [22-25] and for a linear space-charge force it is defined using Eqs. (6) and (9) as

$$
\frac{1}{r\left(Q, \Delta Q_{\mathrm{sc}}\right)}=\frac{1}{r_{0}\left(Q+\Delta Q_{\mathrm{sc}}\right)}-\chi
$$

where $\chi$ is the ratio of the characteristic tune shift [Eq. (6)] to the effective tune spread [Eq. (5)],

$$
\chi=\frac{\Delta Q_{\mathrm{sc}}}{\delta Q_{\mathrm{eff}}}
$$

Using the BTF with space charge [Eq. (10)], we derive a new expression for the pulse response function from the inverse Fourier transform

$g\left(N, \Delta Q_{\text {sc }}\right)=\frac{H(N)}{\pi \delta Q_{\text {eff }}} \int_{0}^{\infty} \cos (2 \pi Q N) \operatorname{Re}\left\{r\left(Q, \Delta Q_{\text {sc }}\right)\right\} \mathrm{d} Q$.

To verify this approach, we perform simulations using the PATRIC code [14]. To produce the results for 2D beams including space charge, the code employs a 2D selfconsistent space charge solver with open boundary conditions and without longitudinal tracking. The number of macroparticles is on the order $10^{6}$ and the number of grid points is $128 \times 128$ for the rectangular calculation domain containing the particles.

A simulation example shows a slower decoherence for stronger space charge (Fig. 2). From the beam offset we extract the oscillation amplitude $A_{\text {beam }}$ (dashed lines in Fig. 2) which is used for further comparisons of the model and simulations. Decoherence for different space charge is presented in a parameter scan in Fig. 3, where the color and the solid lines indicate the simulation results. The dashed lines are given by Eq. (12).

First, we observe the loss of decoherence for $\chi>3$. To explain this effect, we use the stability diagram $\left(1 / r=\Delta Q / \delta Q_{\text {eff }}\right)$ shown in Fig. 4. It is shifted by space charge (the red line) along the real part of $\Delta Q$ [see Eq. (10)]. When the stability diagram is moved away from the coherent tune (in our case the coherent tune is $\Delta Q=0$ ),

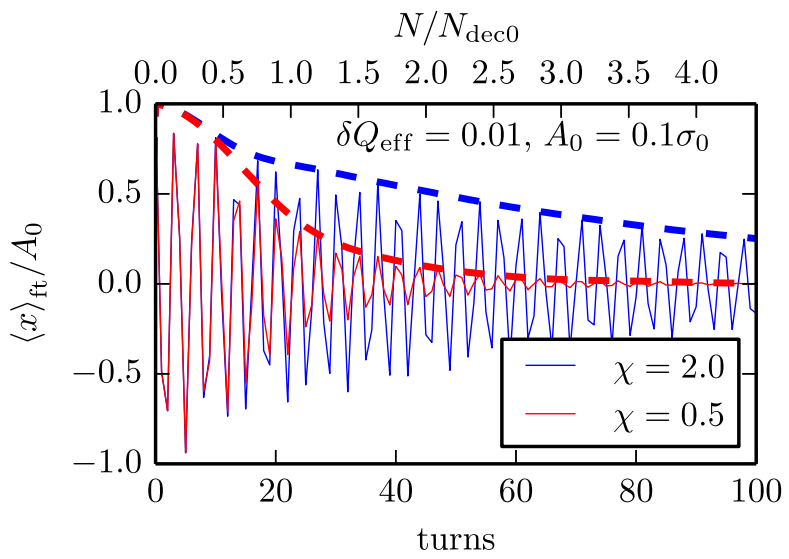

FIG. 2. Transverse decoherence of the kicked beams with space charge from the 2D simulations. The solid lines are the beam offsets and the dashed lines are oscillation amplitudes. 


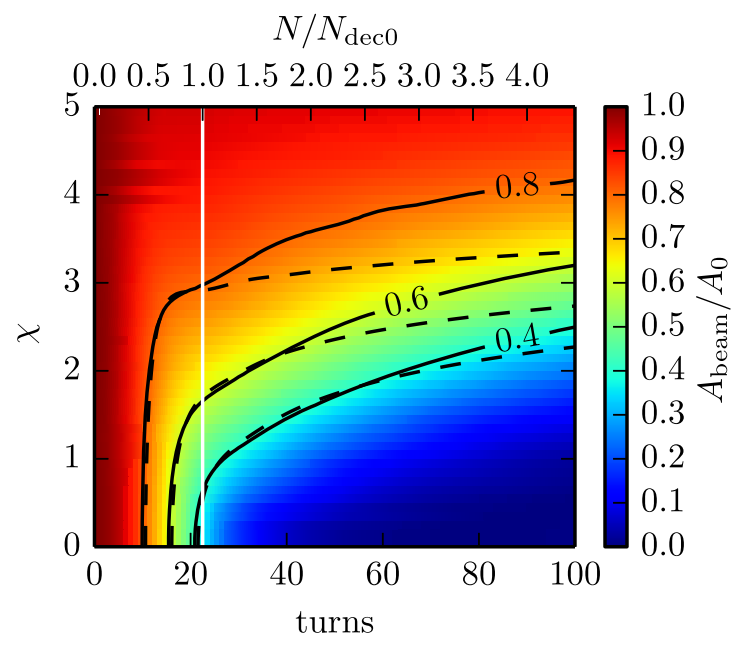

FIG. 3. Time evolution of the beam oscillation amplitude for $2 \mathrm{D}$ beams with different space charge, $\delta Q_{\text {eff }}=0.01$, and $A_{0}=0.1 \sigma_{0}$. Colors and the solid lines (contour lines) are from simulations. Dashed lines are given by Eq. (12). The vertical white line represents $N_{\mathrm{dec} 0}=22.5$ turns [Eq. (8)].

Landau damping is lost. In our simulations we see that it is similar for the loss of decoherence.

Second, in the presented simulation scan the model and simulations agree for $\chi<2$ or for $N<N_{\mathrm{dec} 0}$ (the vertical line in Fig. 3). For stronger space charge decoherence is faster than decoherence obtained from the model. We find that this difference is related to the particle excitation by coherent oscillations of the beam. In simulations we observe that some particles gain large amplitudes. They create a halo around the beam core and-depending on the aperture size - can be lost. The number of lost particles depends on the combination of space charge and the kick strength (Fig. 5). There are no excited particles for $\chi<2$ where decoherence is present. The number of lost particles reaches maximum in the transition region before decoherence is lost and then decreases for stronger space charge. We also see that larger initial kicks lead to stronger

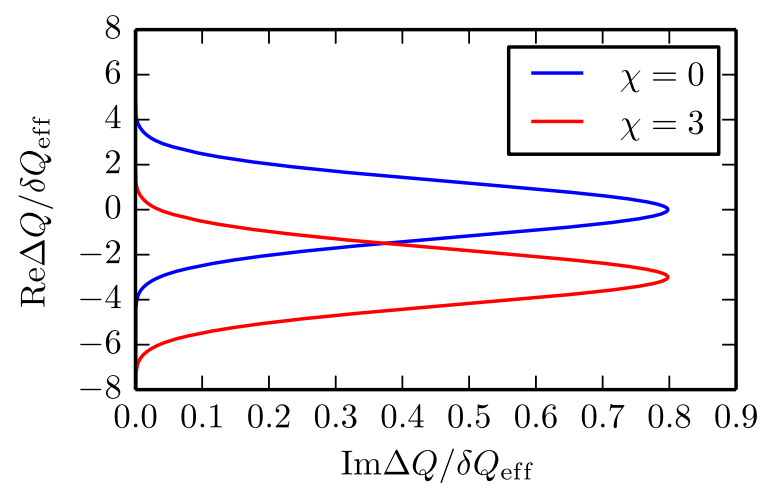

FIG. 4. Stability diagram with and without space charge from Eq. (10).

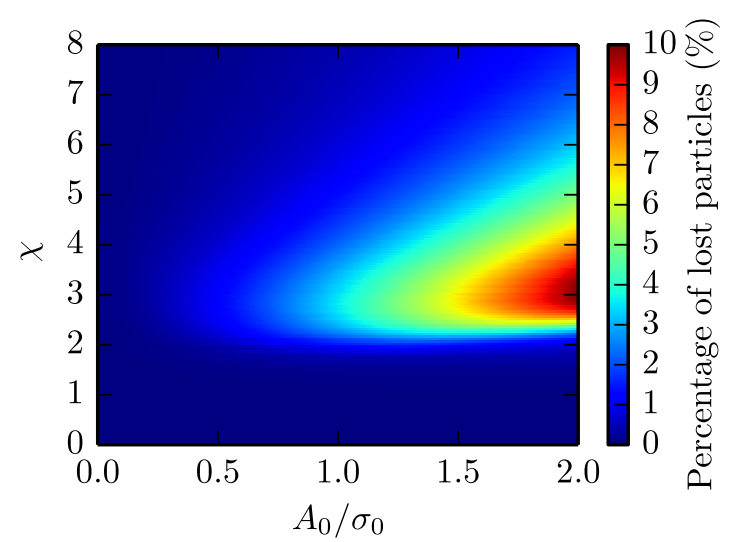

FIG. 5. Beam losses in simulations for the transverse decoherence in $2 \mathrm{D}$ beams with space charge. The particles with the amplitude above $3.5 \sigma_{0}+A_{0}$ are counted as lost particles and are removed from further calculations. The losses saturate after 250 turn. The simulation scan is performed for $\delta Q_{\text {eff }}=0.01$.

losses. All of these observations are in agreement with the excitation in a resonance driven by the space-charge force.

\section{B. Halo buildup due to space charge}

We use a particle-core model to describe halo buildup, similar to Wangler et al. [26]. The equation of motion of the beam centroid $x_{0}$ is

$$
\frac{\mathrm{d}^{2} x_{0}}{\mathrm{~d} N^{2}}+\left(2 \pi Q_{0}\right)^{2} x_{0}=0
$$

We consider the case when decoherence is lost $(\chi>3)$ and the beam continuously oscillates after the initial offset $A_{0}$. The single-particle equation of motion can be modeled as

$$
\frac{\mathrm{d}^{2} x}{\mathrm{~d} N^{2}}+\left(2 \pi Q_{0}\right)^{2}\left(x-F_{\mathrm{sc}}-F_{\xi}\right)=0,
$$

where $x$ is the transverse coordinate of a particle, $F_{\mathrm{sc}}$ is the space-charge force given for a $\mathrm{K}-\mathrm{V}$ transverse distribution with a beam radius $a$,

$$
F_{\mathrm{sc}}= \begin{cases}\frac{2 \Delta Q_{\mathrm{sc}}}{Q_{0}}\left(x-x_{0}\right), & \left|x-x_{0}\right|<a \\ \frac{2 \Delta Q_{\mathrm{sc}}}{Q_{0}} \frac{a^{2}}{\left(x-x_{0}\right)}, & \left|x-x_{0}\right| \geq a\end{cases}
$$

and the additional focusing force is due to chromaticity,

$$
F_{\xi}=\frac{2 \Delta Q_{\xi}}{Q_{0}} x
$$

The tune shift parameters $\Delta Q_{\mathrm{sc}}$ and $\Delta Q_{\xi}$ relate to the single-particle tune $Q=Q_{0}-\Delta Q_{\mathrm{sc}}+\Delta Q_{\xi}$. The particle can be excited if its tune is close to the coherent tune. 


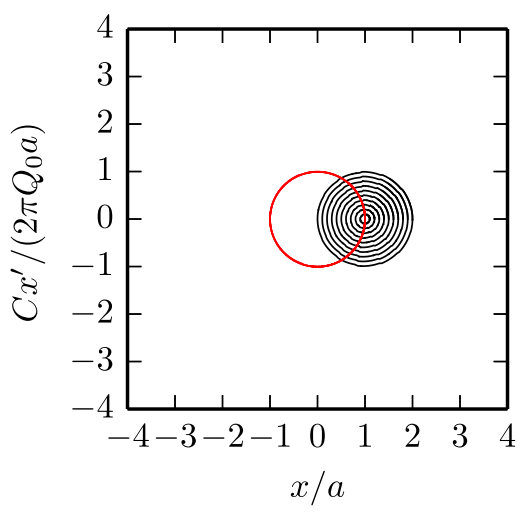

FIG. 6. Stroboscopic plot for 11 particles for two cases: the red points are for zero space charge, and the black points are for zero chromaticity. In the former case, the chromaticity tune shift $\Delta Q_{\xi}$ is in the range of $[-0.03,0.03]$ and all particles have zero initial amplitude with respect to the beam center, $A_{0}=a$. The latter case is for $\Delta Q_{\mathrm{sc}}=0.05$ and different initial offsets in the range $[0, a]$. The plot contains 100 betatron periods.

We solve numerically Eqs. (13) and (14) and present the results using a stroboscopic phase space map of an array of particle trajectories. The stroboscopic method accumulates many snapshots of a phase space taken once per oscillation period. In our case it is taken when the beam offset reaches the maximum value.

The chromaticity alone, and space charge alone, cannot lead to a particle excitation (Fig. 6). All particles with different $\Delta Q_{\xi}$ and $\Delta Q_{\mathrm{sc}}=0$ the amplitude of oscillations does not increase, they just redistribute in phase space. When only space charge is present, all particles remain in the beam core.

The combination of both effects is shown in Fig. 7. We see that some of the particles (4 out of 21) gain amplitude and leave the core. This demonstrates the

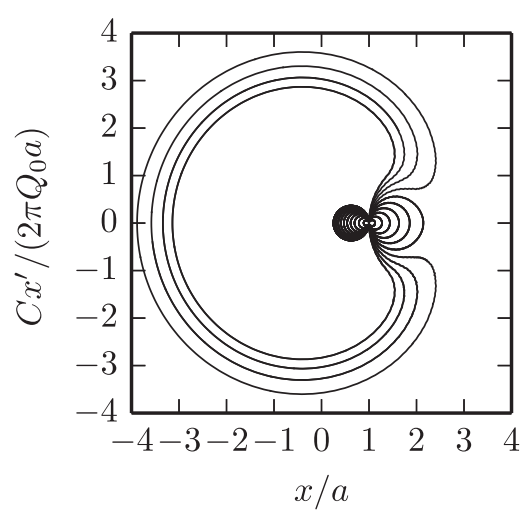

FIG. 7. Stroboscopic plot for 21 particles for the combination of space charge and chromaticity: $\Delta Q_{\xi} \in[-0.03,0.03], \Delta Q_{\mathrm{sc}}=$ 0.05, $Q_{0}=4.29$, and $A_{0}=a$. All particles have zero initial amplitude with respect to the beam center. The plot contains 500 betatron periods. resonant excitation by the space-charge force which we observe in the simulations for coasting beams (Sec. III A).

\section{Bunched beams}

In this section we present an extension of the analytical model [Eq. (12)] to bunched ion beams. We focus on the initial stage of decoherence when the synchrotron motion can be neglected. We consider a bunch as a superposition of independent transverse 2D slices. To calculate the total bunch offset, we sum the offsets of individual slices. Using Eq. (12), we define the pulse response function for a bunch with space charge taking into account the local density $\lambda(z)$,

$$
g_{\mathrm{sc}}\left(N, \Delta Q_{\mathrm{sc}}\right)=\frac{\int_{z_{\min }}^{z_{\max }} g\left[N, \Delta Q_{\mathrm{sc}} \lambda(z) / \lambda_{0}\right] \lambda(z) \mathrm{d} z}{\int_{z_{\min }}^{z_{\max }} \lambda(z) \mathrm{d} z},
$$

where $z$ is the longitudinal coordinate. For the systematic study of decoherence in bunches we introduce the spacecharge parameter and the effective parameter using Eqs. (5) and (6)

$$
q_{\mathrm{sc}}=\frac{\Delta Q_{\mathrm{sc}}}{Q_{s}} \quad \text { and } \quad q_{\mathrm{eff}}=\frac{\delta Q_{\mathrm{eff}}}{Q_{s}} .
$$

To obtain the applicability region of the extended model [Eq. (17)], we perform simulations with the PATRIC code. For space charge calculations in bunches, the " $2.5 \mathrm{D}$ space charge" approach is employed [14]. Every time step the bunch is divided longitudinally into 2D slices, for each slice, a 2D self-consistent space charge solver is used to obtain the distribution of electric fields.

In the simulation example without space charge, the beam oscillation amplitude corresponds to alternating decoherence and recoherence processes (Fig. 8). With space charge, decoherence is slower, recoherence is not related to $Q_{s}$, and after the transition time we have the

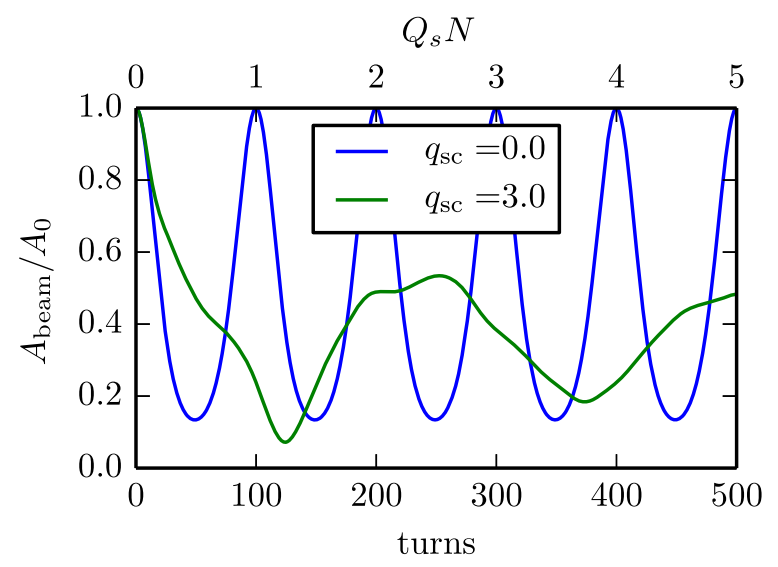

FIG. 8. Time evolution of the oscillation amplitude from simulations for bunches with Gaussian transverse and longitudinal distributions. Simulation parameters: $q_{\mathrm{eff}}=1, A_{0}=\sigma_{0}$, $Q_{s}=0.01$. 


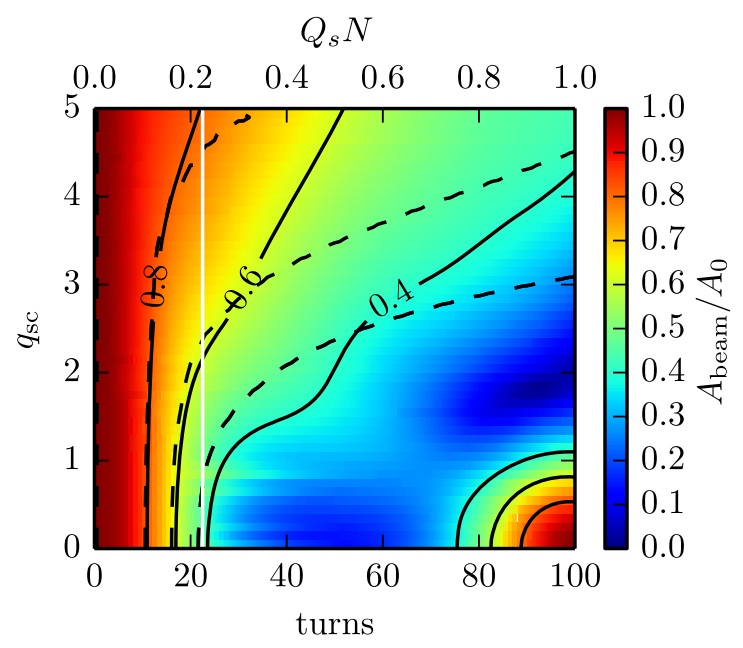

FIG. 9. Decoherence in bunches with space charge. Colors and solid lines are from simulations for bunches. Dashed contour lines are given by Eq. (17). The vertical white line is $N_{\mathrm{dec} 0}=22.5$ turns [Eq. (8)]. Simulation parameters: $q_{\mathrm{eff}}=1$, $A_{0}=\sigma_{0}, Q_{s}=0.01$.

beating of the remaining head-tail modes [13]. Decoherence of bunches for different space charge is presented in a parameter scan in Fig. 9, where the color and the solid lines indicate the simulation results, and the dashed lines are given by Eq. (17). The model agrees with simulations for $N<N_{\operatorname{dec} 0}$ (the white vertical line) as it is for coasting beams (Fig. 3). For $N>N_{\operatorname{dec} 0}$, decoherence in simulations is faster than decoherence from the model. The first reason is that the resonant excitation of the particles leads to fast losses (Fig. 10). The number of lost particles depends on the combination of different parameters $\left(q_{\mathrm{sc}}\right.$, $q_{\mathrm{eff}}, A_{0}$, and the aperture radius) which is not studied here in detail. The second reason for the faster decoherence in

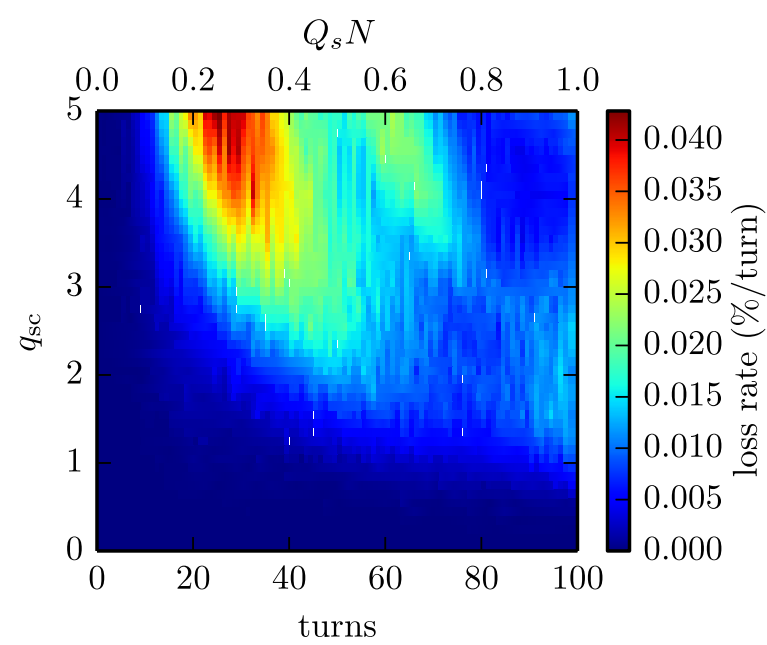

FIG. 10. Loss rate for different space charge from the simulation scan shown in Fig. 9. The aperture radius is $3.5 \sigma_{0}+A_{0}$.

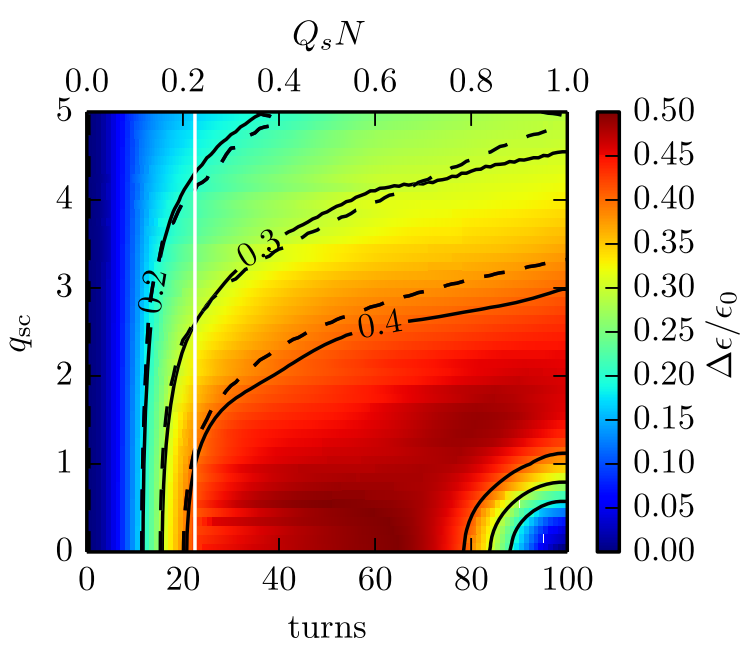

FIG. 11. Time evolution of the emittance growth for decoherence with space charge shown in Fig. 9. Colors and solid lines are from simulations of bunches. Dashed lines are calculated using Eq. (3) from the oscillation amplitude given by Eq. (17). The vertical white line is $N_{\operatorname{dec} 0}=22.5$ turns [Eq. (8)].

simulations is the synchrotron motion. The synchrotron motion produces an additional mixing of particles which is not included in the model.

Without space charge, the emittance growth can be predicted from the beam oscillation amplitude using Eq. (3). To obtain evolution of emittance with space charge, we use Eq. (3) in combination with Eq. (17). For the simulation scan shown in Fig. 9, we plot the rms emittance in Fig. 11. Without space charge, we observe emittance growth for $N<N_{s} / 2$ and then the emittance returns to the initial value after the synchrotron period because of compensation of the betatron phase spread. With space charge, the emittance calculated from Eq. (3) and Eq. (17) sufficiently reproduces the simulation results. The emittance blowup is smaller for stronger space charge during the first synchrotron period.

The pulse response function, by definition, does not depend on the initial oscillation amplitude, but the maximum emittance blowup is directly defined by $A_{0}$ [see Eq. (4)]. We compare decoherence for different $A_{0}$ in Fig. 12. Simulations with $q_{\mathrm{sc}}=4$ for $A_{0}=0.5 \sigma_{0}$ and $A_{0}=1.0 \sigma_{0}$ are almost identical and agree with the model (the dashed line) for $N<N_{\operatorname{dec} 0}$ (the left-hand side plot in Fig. 12). Decoherence is a bit faster due to stronger losses for $A_{0}=2 \sigma_{0}$. The situation for the emittance is similar. The analytical approach [Eq. (3) in combination with Eq. (17)] reproduces the simulations for $N<N_{\operatorname{dec} 0}$ (the right-hand side plot in Fig. 12).

We find in simulations that the characteristic decoherence time $N_{\mathrm{dec}}$ in bunches depends on $q_{\mathrm{sc}}$ and $q_{\text {eff }}$. A simulation scan for different combinations of these parameters for $A_{0}=\sigma_{0}$ is shown in Fig. 13. Decoherence is slower for stronger space charge and the decoherence time 

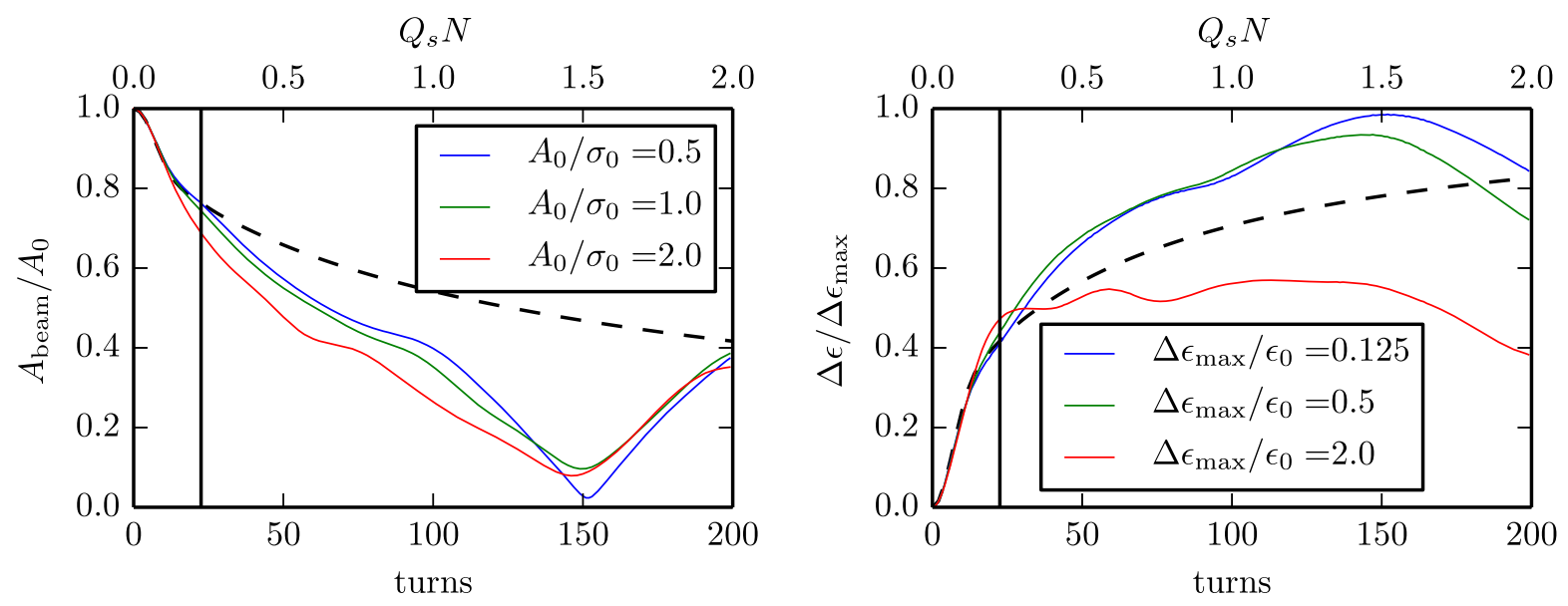

FIG. 12. Decoherence for different initial kick strength. The left-hand side plot is the time evolution of the beam oscillation amplitude. The dashed line is given by Eq. (17). The right-hand side plot is the time evolution of the emittance growth. The dashed line is calculated using Eq. (3) from the oscillation amplitude given by Eq. (17). The vertical black line is $N_{\text {dec0 }}=22.5$ turns [Eq. (8)]. Simulation parameters: $q_{\mathrm{eff}}=1, Q_{s}=0.01$.

can exceed the synchrotron period (the dark-red region). For larger effective spread we have a faster decoherence.

In this discussion the decoherence and the emittance growth are governed by the interplay of chromaticity and space charge which slows down these processes. However, the initial stage of decoherence in bunched beams can be also modified by impedances.

\section{INFLUENCE OF IMAGE CHARGES}

We consider the effect of imaginary impedances which produce coherent tune shifts. For the case of a circular pipe with radius $b$ and a round beam with radius $a=2 \sigma_{0}$, the coherent tune shift due to image charges is

$$
\Delta Q_{\mathrm{coh}}=\Delta Q_{\mathrm{sc}} \frac{a^{2}}{b^{2}}
$$

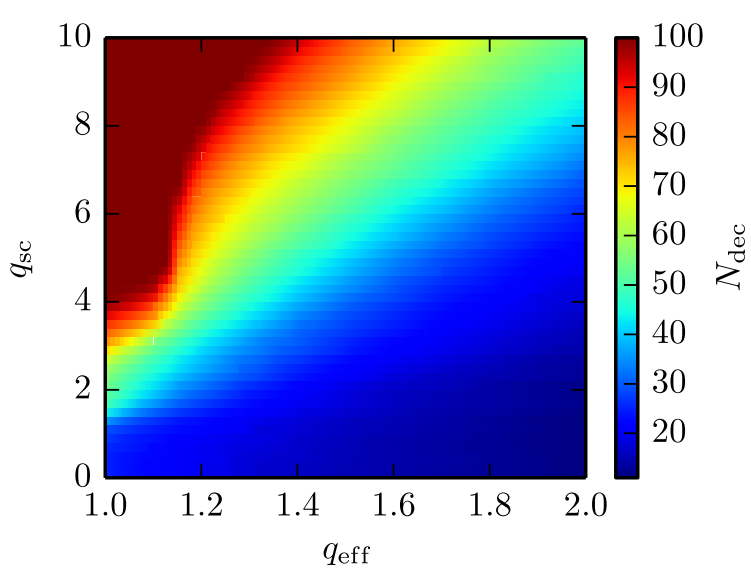

FIG. 13. The decoherence time $N_{\text {dec }}$ for difference space charge and the effective tune spread. Simulation parameters: $A_{0}=\sigma_{0}, Q_{s}=0.01$.
The role of image charges in the decoherence process of coasting beams can be understood using the stability diagram. The coherent tune shift moves the origin in Fig. 4 towards the stability diagram shifted by space charge. Hence, image charges partly compensate the effect of space charge and can restore Landau damping.

To illustrate the role of image charges in ion bunches, we consider the situation with $q_{\mathrm{sc}} \gg q_{\mathrm{eff}}$ and $q_{\mathrm{sc}} \gg q_{\mathrm{coh}}$, where the image-charge parameter is

$$
q_{\mathrm{coh}}=\frac{\Delta Q_{\mathrm{coh}}}{Q_{s}} .
$$

In this case decoherence is lost for the majority of longitudinal slices in the bunch. Image charges produce a local coherent tune shift which is different for each slice. Thus decoherence is present because of the coherent tune spread which mixes betatron phases of different slices. To describe the initial stage of decoherence in the presence of image charges, we neglect the synchrotron motion and consider a bunch as a superposition of longitudinal slices with different coherent tune shifts. We obtain the pulse response function as

$$
g_{\mathrm{ic}}\left(N, \Delta Q_{\mathrm{coh}}\right)=\frac{\int_{z_{\min }}^{z_{\max }} \cos [2 \pi N Q(z)] \lambda(z) \mathrm{d} z}{\int_{z_{\min }}^{z_{\max }} \lambda(z) \mathrm{d} z},
$$

where $Q(z)=Q_{0}-\Delta Q_{\text {coh }} \lambda(z) / \lambda_{0}$ is the local coherent tune.

To verify this approach, we perform simulations using the PATRIC code. Additionally to the simulation model discussed in Sec. III C, we include the coherent tune shift caused by image charges in the round pipe via impedance kicks [14]. Decoherence for different space charge and image charges is presented in a parameter scan in Fig. 14, 


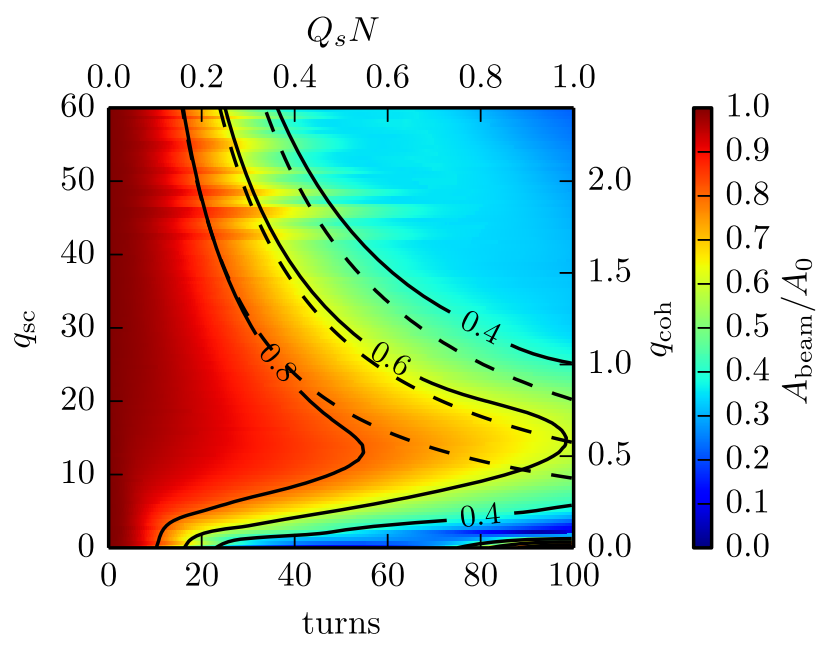

FIG. 14. Decoherence in bunches with space charge and image charges. Colors and solid lines are from simulations. Dashed lines are given by Eq. (21). Simulation parameters: $a / b=1 / 5$, $q_{\mathrm{eff}}=1, A_{0}=\sigma_{0}, Q_{s}=0.01$.

where colors and the solid lines indicate the simulation results, and the dashed lines are given by Eq. (21). For $q_{\mathrm{coh}}<0.5$, the coherent tune spread is small and decoherence is dominated by interplay of chromaticity and space charge. For $q_{\text {coh }}>1$, the process is governed by image charges and can be sufficiently reproduced by the model [Eq. (21)].

In general, it is necessary to compare the space charge tune shift, the coherent tune shift, and the effective tune spread, in order to understand which effect dominates the initial stage of the decoherence process. For $q_{\text {coh }}<0.5$, image charges play no role. For the case where $q_{\text {coh }} \approx 1$, decoherence is governed by interplay of chromaticity, space charge and image charges. When the conditions $q_{\mathrm{sc}} \gg q_{\text {eff }}$ and $q_{\mathrm{coh}}>1$ are fulfilled, decoherence is dominated by image charges.

\section{MEASUREMENTS AND COMPARISONS}

We performed the decoherence measurements for intense ion beams in the SIS18 synchrotron [15] at GSI Darmstadt. Bunches of $\mathrm{Ni}_{58}^{26+}$ ions were stored at the kinetic energy of $100 \mathrm{MeV} / \mathrm{u}$ and kicked transversally with the kick duration of one turn. The bunch offset signals were recorded using BPMs. SIS18 general parameters and settings in our experiments were $C=216.72 \mathrm{~m}$, $\eta=-0.79, \quad Q_{0, x}=4.29, \quad Q_{0, y}=3.27, \quad \xi_{x}=-1.3$, $\xi_{y}=-2.1, Q_{s}=4.1 \times 10^{-3}$, and the harmonic $h=4$. The number of particles per beam was between $N_{p}=$ $1.6 \times 10^{9}$ and $N_{p}=4 \times 10^{9}$. An example of the bunched beam decoherence in the horizontal plane is shown in Fig. 15. We see that decoherence with space charge is slower than the synchrotron period and the later stage is the beating of the residual head-tail modes.

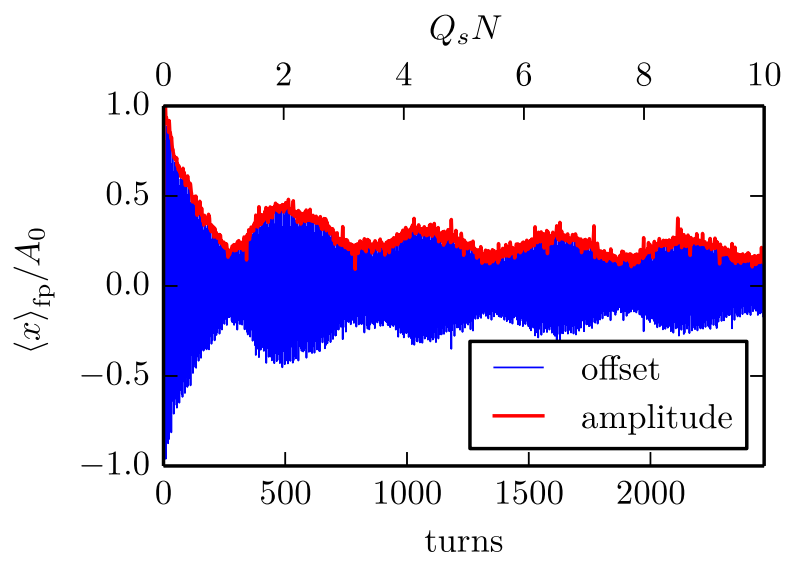

FIG. 15. Time evolution of the bunch offset in the horizontal plane from BPM measurements in SIS18 after a transverse kick. One turn corresponds to $1.682 \mu \mathrm{s}, A_{0} \approx 4 \mathrm{~mm}$.

The possible contribution of the transverse nonlinearities was checked by the comparisons of decoherence for the optimized and for the distorted closed orbit. In our measurements in the SIS18 synchrotron, the initial stage does not differ and the residual oscillations have similar amplitudes.

As we discussed in the previous section, image charges can change the initial stage of the decoherence process. We estimate the maximum value of the parameter $q_{\text {coh }}$ taking average radii of the SIS18 beam pipe for the horizontal and the vertical planes: $b_{x}=100 \mathrm{~mm}$ gives $q_{\mathrm{coh}, x}=0.05$, and $b_{y}=70 \mathrm{~mm}$ gives $q_{\mathrm{coh}, y}=0.15$. Image charges can be neglected for our measurements according to the simulation study in Sec. IV.

Decoherence can also be modified by rf nonlinearities, which are especially important for the later stage of decoherence and recoherence processes [13]. Simulations with realistic rf voltage showed that rf nonlinearities can be neglected for the initial stage of decoherence.

We show that in our measurements the decoherence was governed by chromaticity and space charge. We see a slower decoherence for stronger space charge from the measured signals for the same machine settings and different intensities (Fig. 16). To estimate the space-charge parameter, we fit the decoherence signals from the simulations with the input parameters $q_{\mathrm{eff}}$, and $Q_{s}$ taken from the measurements. For the fitting process, we use the signals during the first synchrotron period. The spacecharge parameter can be also estimated from the measured emittance. We take into account the elliptical cross section by substituting $\epsilon$ in Eq. (6) by $\left(\epsilon_{x}+\sqrt{\epsilon_{x} \epsilon_{y} Q_{0, x} / Q_{0, y}}\right) / 2$. For the case of higher intensity (the red line) the emittances are $\epsilon_{x}=10 \mathrm{~mm} \mathrm{mrad}$ and $\epsilon_{y}=2.5 \mathrm{~mm} \mathrm{mrad}$. The estimated parameter $q_{\mathrm{sc}} \approx 5$ is almost 2 times smaller than the fitted value from the simulation. The difference is due to the uncertainty in the transverse beam size measurements at SIS18 which was also the case in previous works $[13,25]$. 


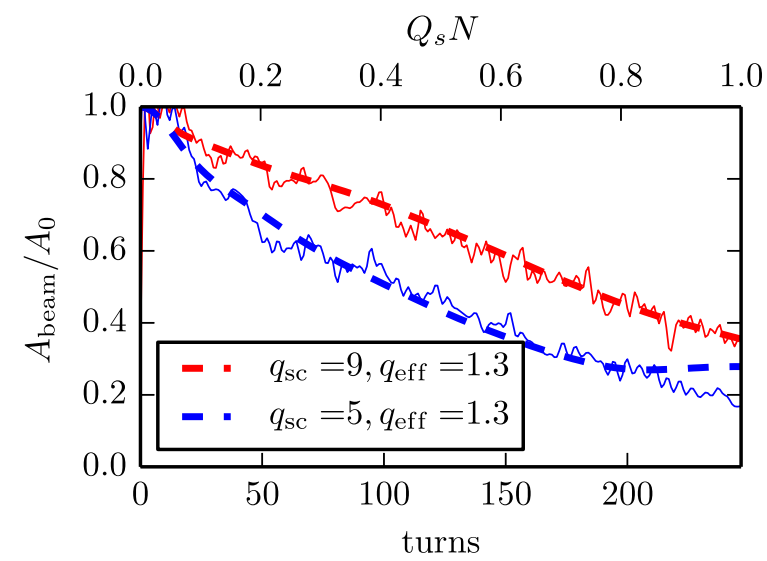

FIG. 16. Decoherence with space charge from the measurements: the solid red line is for $N_{p}=4 \times 10^{9}$, the blue solid line is for $N_{p}=1.6 \times 10^{9}$. The dashed lines are from simulations with the fitted $q_{\mathrm{sc}}$.

In simulations we set $A_{0}=\sigma_{0}$ which corresponds to the case when the decoherence does not depend on the initial kick (see Fig. 12). This agrees with measurements where we also observe a weak dependence of decoherence on the kick strength (Fig. 17). Only for the strongest kick (the black line) decoherence is a bit faster.

The role of chromaticity is shown in Fig. 18 where for the same beam settings we compare the signals for the natural $\left(\xi_{x}=-1.3, q_{\text {eff }}=1.3\right)$ and the compensated chromaticity $\left(\xi_{x} \approx 0, q_{\text {eff }}=0.5\right.$ ). As for the case without space charge, decoherence is slower for a smaller effective parameter $q_{\text {eff }}$. For $q_{\mathrm{sc}} \gg q_{\mathrm{eff}}$, the oscillation amplitude practically does not decrease during the first synchrotron period due to the loss of decoherence.

Finally, the summary of the measurements is shown in Fig. 19. The points are the characteristic decoherence time $N_{\mathrm{dec}}$ from the measured signals. Solid lines are from the simulation scan for $A_{0}=\sigma_{0}$ which was similar with the estimated kick strength in measurements. Decoherence for

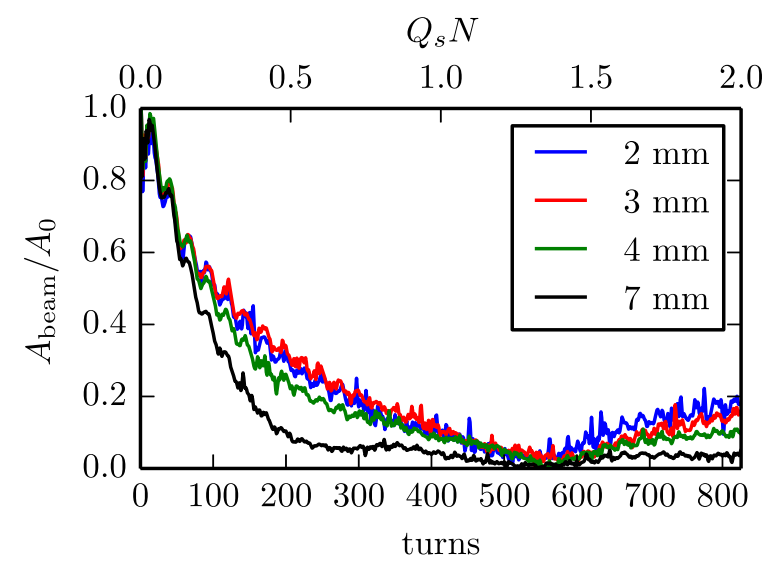

FIG. 17. Decoherence with space charge from measurements in SIS18 for different kick strength.

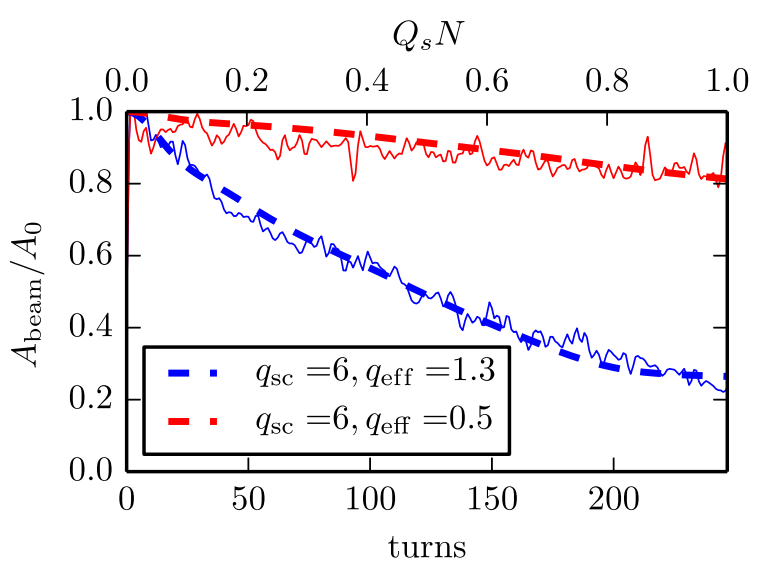

FIG. 18. Decoherence with space charge from measurements in SIS18: the solid blue line for the natural chromaticity $\left(\xi_{x}=-1.3\right.$, $q_{\text {eff }}=1.3$ ), the solid red line for the compensated chromaticity $\left(\xi_{x} \approx 0, q_{\text {eff }}=0.5\right)$. The dashed lines are from simulations with the fitted $q_{\mathrm{sc}}$.

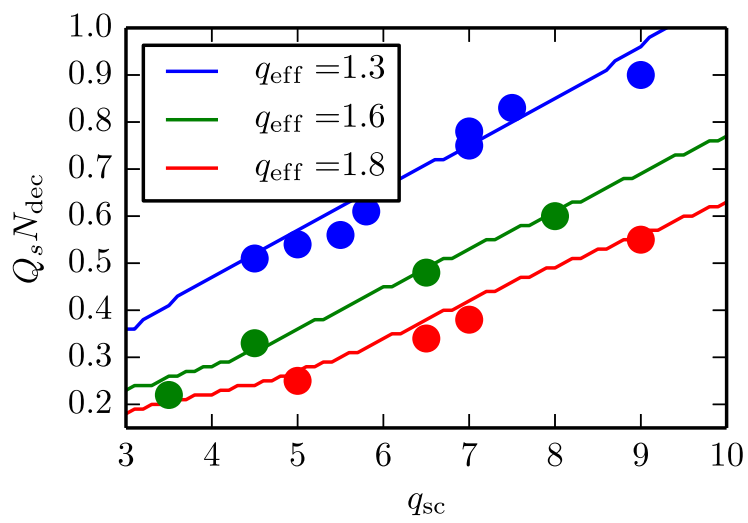

FIG. 19. Summary of the decoherence measurements in SIS18. The points correspond to the decoherence time obtained from the measured signals for different machine and beam parameters. Lines are the characteristic decoherence time from simulations.

larger space-change parameter is slower. For higher effective parameter $q_{\text {eff }}$ we observe a faster decoherence.

\section{CONCLUSIONS}

The initial stage of decoherence determines the transverse emittance blowup of injected (or kicked) bunches in synchrotrons. A detailed understanding of the transverse decoherence for intense beams is necessary to determine the feedback system requirements in synchrotrons with the goal to preserve the beam quality.

In the present work decoherence is studied with a focus on interplay of chromaticity, space charge, and image charges for bunched beams. Without intensity effects the relation of the transverse emittance and the oscillation amplitude following an arbitrary initial offset is given by Eq. (3). We find that the same expression can be used for 
intense ion bunches with a modified beam oscillation amplitude [Eqs. (17) and (21)] during the initial stage of the decoherence process.

For a coasting beam we showed in particle tracking simulations (Fig. 3) and in our analytical model [Eq. (12)] that the addition of space charge in the pulse response function leads to the loss of decoherence in cases of strong space charge $\left(\Delta Q_{\mathrm{sc}}>3 \delta Q_{\text {eff }}\right)$. Especially in the initial stage of decoherence the analytical model agrees very well with the particle tracking simulations and later deviations can be explained by beam losses and beam halo buildup due to resonant excitation of particles. The latter was illustrated in particle tracking simulations (Fig. 5) and in a particle-core approach (Sec. III B), where we observed the excitation driven by the coherent oscillations of the beam. The particles are in resonance when the incoherent tune is close to the coherent tune $\left(Q \approx Q_{0}-\Delta Q_{\text {coh }}\right)$.

We extended our 2D approach to bunched ion beams as a longitudinally sliced model, with different analytical expressions [Eqs. (17) and (21)] depending on the chromaticity tune spread, space charge, and image charges. These descriptions agree well in all regimes in the initial stage of decoherence with self-consistent particle tracking simulations (Figs. 9 and 14). In general, there is a slower decoherence for stronger space charge. Similar to the 2D beam decoherence can be lost in longitudinal slices of the bunched beam for large space-charge parameters $q_{\mathrm{sc}} \gg q_{\text {eff }}$. However, decoherence can still be restored by image charges in the beam pipe due to the coherent tune spread.

Finally, we showed results of the measurements in the SIS18 synchrotron at GSI Darmstadt in comparison with our particle tracking simulations. The systematic behavior of the decoherence process shows that the agreement between the measurements and the particle tracking simulations is good with the space charge parameter as a free fitting parameter. The observed difference is due to uncertainty in the transverse beam size measurements which was also the case in previous works. The main contributors to the initial stage of decoherence process can be identified as chromaticity and space charge in the case of our measurements in SIS18; nonlinearities and image charges play a minor role.

[1] I. C. Hsu, The decoherence and recoherence of the betatron oscillation signal and an application, Part. Accel. 34, 43 (1990).

[2] R. E. Meller, A. W. Chao, J. M. Peterson, S. G. Peggs, and M. Furman, SSC Report No. SSC-N-360, 1987.

[3] M. Minty, A. Chao, and W. Spence, in Proceedings of the Particle Accelerator Conference, Dallas, TX, 1995 (IEEE, New York, 1995), p. 3037.
[4] M. Reiser, Free energy and emittance growth in nonstationary charged particle beams, J. Appl. Phys. 70, 1919 (1991).

[5] L. Vos, Report No. CERN-SL-98-056 AP, 1998.

[6] J. Belleman, A. Blas, J.-L. Gonzalez, F. Caspers, W. Hofle, T. Kroyer, R. Louwerse, F. Pedersen, V. Rossi, and J. Tuckmantel, CERN Report No. AB-Note-2005-027, 2005.

[7] A. Blas, J. Belleman, E. Benedetto, F. Caspers, D. Glenat, R. Louwerse, M. Martini, E. Métral, V. Rossi, and J. Sladen, in Proceedings of the 23rd Particle Accelerator Conference, Vancouver, Canada, 2009 (IEEE, Piscataway, 2009), p. 2183.

[8] I. Karpov, V. Kornilov, and O. Boine-Frankenheim, in Proceedings of the 54th ICFA Advanced Beam Dynamics Workshop on High-Intensity, High Brightness and High Power Hadron Beams, East Lansing, MI, USA (JACoW, CERN, Geneva, 2015), pp. 45-49.

[9] G. Stupakov and A. Chao, SSC Report No. SSCL-621, 9, 1993.

[10] G. Stupakov and A. Chao, in Proceedings of the Particle Accelerator Conference, Dallas, TX, 1995 (IEEE, New York, 1995), p. 3288.

[11] O. Boine-Frankenheim, in Proceedings of the International Particle Accelerator Conference, Kyoto, Japan (ICR, Kyoto, 2010), p. 2430.

[12] V. Kornilov and O. Boine-Frankenheim, in Proceedings of the 46th ICFA Advanced Beam Dynamics Workshop on High-Intensity and High-Brightness Hadron Beams, Morschach, Switzerland (JACoW, CERN, Geneva, 2011), pp. 101-105.

[13] V. Kornilov and O. Boine-Frankenheim, Transverse decoherence and coherent spectra in long bunches with space charge, Phys. Rev. ST Accel. Beams 15, 114201 (2012).

[14] O. Boine-Frankenheim and V. Kornilov, in Proceedings of the 9th International Computational Accelerator Physics Conference, Chamonix Mont-Blanc, France (JACoW, CERN, Geneva, 2006), pp. 267-270.

[15] K. Blasche and B. Franczak, in Proceedings of the 3rd European Particle Accelerator Conference, Berlin, Germany (Editions Frontieres, Gif-sur-Yvette, 1992), pp. $9-13$.

[16] V. Kornilov and O. Boine-Frankenheim, in Proceedings of the 10th International Computational Accelerator Physics conference, San Francisco, CA, USA (JACoW, CERN, Geneva, 2009), pp. 58-63.

[17] A. W. Chao and M. Tigner, in Handbook of Accelerator Physics and Engineering (World Scientific, Singapore, 1998), p. 91.

[18] D. A. Edwards and M. J. Syphers, An Introduction to the Physics of High Energy Accelerators (Wiley-VCH Verlag GmbH, Weinheim, Germany, 2004), p. 304.

[19] K.-Y. Ng, Physics of Intensity Dependent Beam Instabilities (World Scientific, Singapore, 2006), p. 105.

[20] A. Hofmann, CERN Accelerator School, Report No. CERN-1995-006, 275, 1995.

[21] A. W. Chao, Physics of Collective Beam Instabilities in High Energy Accelerators (John Wiley \& Sons, Inc., New York, 1993), p. 236. 
[22] D. Pestrikov, Dipole coherent oscillations and fluctuations of a coasting ion beam with strong space charge, Nucl. Instrum. Methods Phys. Res., Sect. A 562, 65 (2006).

[23] D. Pestrikov, Self-consistent dipole coherent oscillations of a coasting ion beam with strong space charge, Nucl. Instrum. Methods Phys. Res., Sect. A 578, 65 (2007).

[24] O. Boine-Frankenheim, V. Kornilov, and S. Paret, Measurement and simulation of transverse Schottky noise with space charge, Phys. Rev. ST Accel. Beams 11, 074202 (2008).

[25] S. Paret, V. Kornilov, O. Boine-Frankenheim, and T. Weiland, Transverse Schottky and beam transfer function measurements in space charge affected coasting ion beams, Phys. Rev. ST Accel. Beams 13, 022802 (2010).

[26] T. P. Wangler, K. R. Crandall, R. Ryne, and T. S. Wang, Particle-core model for transverse dynamics of beam halo, Phys. Rev. ST Accel. Beams 1, 084201 (1998). 